\title{
Wer selektioniert sich in die Ausbildung zur Sekundarlehrperson? Studienwahl vor dem Hintergrund sozialer Herkunft, pädagogischer Interessen und fachlicher Orientierung
}

\section{Andrea Keck Frei, Simone Berweger, Stefan Denzler, Christine Bieri Buschor und Christa Kappler}

Dieser Beitrag geht der Frage nach, wer sich in Lehramtsstudiengänge selektioniert und ob Unterschiede zwischen angehenden Lehrpersonen der Primarstufe und der Sekundarstufe I festgestellt werden können. Dabei zeigt sich zum einen, dass sich Interessenten und Interessentinnen für Lehramtsstudiengänge in Bezug auf die soziale Herkunft, die Leistung (Deutschnote) und ihre Zeitpräferenz von den übrigen Maturanden und Maturandinnen unterscheiden. Zum anderen erweisen sich angehende Lehrpersonen der Sekundarstufe I als stärker fachorientiert und weniger pädagogisch interessiert als angehende Lehrpersonen der Primarstufe.

\section{Einleitung und Fragestellung}

Vor der Reform der Schweizer Lehrerinnen- und Lehrerbildung in den 1990erJahren absolvierten Lehrpersonen in der Schweiz nach Unterrichtsstufe, Schultyp und Anspruchsniveau getrennt organisierte Ausbildungsgänge: Volksschullehrkräfte $^{1}$ der Primarstufe sowie Lehrkräfte für Schultypen der Sekundarstufe I mit Grundanforderungen wurden in je separaten Programmen zu Allroundern an Lehrerseminarien ausgebildet. Lehrpersonen für Schultypen der Sekundarstufe I mit erweiterten Anforderungen absolvierten einen Teil ihrer fachlichen Ausbildung an einer Universität, Gymnasiallehrkräfte durchliefen nach einem universitären Fachstudium eine postgraduale pädagogische und fachdidaktische Ausbildung an der Universität. Seit der Reform werden Volksschullehrpersonen gesamthaft an pädagogischen Hochschulen (PH) auf Fachhochschulniveau ausgebildet. Primarlehrkräfte erlangen einen breit gefächerten Bachelor-Abschluss. Die mit Master-Abschluss organisierte Ausbildung von Lehrkräften der Sekundarstufe I erfolgt heute unabhängig vom angestrebten Anspruchsniveau, ist stärker fachlich ausgerichtet und wird teilweise in Kooperation mit Universitäten angeboten. Die frühere Situation zeichnete sich dadurch aus, dass die institutionell und organisatorisch verschiedenen Ausbildungsgänge Studierende mit unterschiedlichen motivationalen Orientierungen anzogen. Die Ausbildung 
erfolgte mit engem Bezug auf die spätere Berufstätigkeit, d.h. mit einem starken Fokus auf die unterschiedlichen kognitiven und sozialen Voraussetzungen der Schülerinnen und Schüler. Heute sind die Studiengänge an den pädagogischen Hochschulen allgemeiner ausgerichtet; differenziert wird primär zwischen den beiden Unterrichtsstufen Primar- und Sekundarstufe I. Die stärkere Vereinheitlichung der Bachelor- und Masterstudiengänge für Lehrkräfte der Volksschule zielte u.a. auch darauf ab, international anerkannte Abschlüsse zu gewährleisten sowie neue Weiterbildungs- und Laufbahnoptionen für den Lehrberuf zu erschliessen. Dadurch sollte der Lehrberuf an Attraktivität gewinnen (Criblez, 2010; EDK, 2008; Leutwyler, 2007).

Die spezielle Herausforderung der Ausbildung für die Sekundarstufe I zeigt sich darin, dass sie den Aufbau einer breiten Palette an Kompetenzen verlangt, um den Bedürfnissen der Lernenden der Schultypen mit verschiedenen Anspruchsniveaus gerecht zu werden. Angesichts der Vereinheitlichung der verschiedenen Studiengänge an pädagogischen Hochschulen stellt sich die Frage, inwiefern sich Studierende im Studiengang der Sekundarstufe I von anderen Studierendengruppen systematisch unterscheiden. Während früher die Selbstselektion in die institutionell unterschiedlichen Institutionen auf der Hand lag, ist dies heute mit der Ansiedelung aller Ausbildungsgänge für die Volksschullehrkräfte an pädagogischen Hochschulen nicht mehr unbedingt gegeben. Die Frage nach allfälligen Unterschieden zwischen den Studierenden hinsichtlich Studienwahlmotive sowie sozialer und persönlicher Merkmale ist jedoch für die pädagogischen Hochschulen für die Organisation der Ausbildung ebenso wie für die Bildungspolitik im Zusammenhang mit aktuellen Fragen zur Rekrutierung, Aus- und Weiterbildung sowie zum Verbleib im Lehrberuf von hoher Relevanz. Um den Aspekt der Selbstselektion bei der Berufs- und Studienwahl von Lehrkräften nicht nur als individuellen Laufbahnentscheidungsprozess zu analysieren, sondern diesen auch unter der gesellschaftlichen Perspektive zu beleuchten, werden im Folgenden neben psychologischen auch soziologische Theorien zu Bildungsentscheidungen herangezogen.

\section{Theorie und Forschungsstand}

Studienwahlentscheide sind in Ländern wie der Schweiz, in denen sich die formalen Zulassungsbedingungen zu universitären Hochschulen mit Ausnahme des Medizinstudiums ${ }^{2}$ auf das Vorliegen eines gymnasialen Maturitätsabschlusses beschränken, ausschliesslich das Ergebnis von Prozessen der Selbstselektion. Darunter verstehen wir individuelle Wahlentscheide zwischen verschiedenen Ausbildungsoptionen auf der Grundlage von Faktoren wie der sozialen Herkunft, Interessen, Fähigkeiten und Werthaltungen. Die Präferenz für den Studiengang der Sekundarstufe I an einer pädagogischen Hochschule wird folglich von Individuen mit ganz bestimmten Merkmalen geäussert. Es 
ist diese «differenzielle Anreizwirkung» unterschiedlicher Angebote, welche den Prozess der Selbstselektion bestimmt (Luhmann \& Mayntz, 1973, S. 23). Selbstselektive Prozesse prägen die Zusammensetzung der Studierenden unterschiedlicher Studiengänge und Hochschultypen. Daher sind die Faktoren, die auf diese Wahlprozesse einwirken, von besonderer Relevanz für eine erfolgsversprechende Organisation der Ausbildung und eine nachhaltige Rekrutierung zukünftiger Lehrpersonen.

Zur Erklärung von selbstselektiven Bildungsentscheidungen werden Rational-Choice- bzw. Wert-Erwartungstheorien herangezogen (Boudon, 1973, 1987; Breen \& Goldthorpe, 1999; Coleman, 1994; Erikson \& Jonsson, 1996; Esser, 1993). Laut diesen Ansätzen wägen Individuen beim Entscheid für eine bestimmte Ausbildung ihre subjektiven Einschätzungen von antizipierten Kosten (monetärer sowie nicht-monetärer Art), Nutzen und Erfolgswahrscheinlichkeit der verschiedenen Ausbildungsoptionen gegeneinander ab. In der soziologischen Tradition steht die soziale Selektivität von Bildungskarrieren im Vordergrund (Boudon, 1973; Bourdieu \& Passeron, 1970). Dabei spielen unterschiedlich verfügbare Ressourcen sowie familiär bedingte Bildungs- und Statusaspirationen eine zentrale Rolle. Diese schichtspezifische Ausgangssituation erklärt die unterschiedlichen Bildungsentscheidungen verschiedener sozialer Gruppen (Boudon, 1987; Erikson \& Jonsson, 1996).

In der Berufswahl- und Laufbahnpsychologie steht eine möglichst gute Passung zwischen den individuellen Merkmalen einer Person und den Anforderungen der beruflichen Umwelt im Zentrum (Holland, 1997). Individualpsychologische Theorien fokussieren insbesondere Fähigkeiten, Interessen und Erwartungen sowie das individuelle Bedürfnis, diese in der Berufs- und Studienwahl möglichst optimal zu realisieren und mit Aufgaben aus anderen Lebensbereichen zu vereinbaren (Dawis \& Lofquist, 1984; Eccles, 1983, 1994; Gottfredson, 1981; Holland, 1997; Super, 1980). Erweist sich die Passung als mangelhaft, kann dies zu einem Ausbildungsabbruch, einem Studienfachwechsel oder zum Verlassen der eingeschlagenen Laufbahn führen. Die sozial-kognitive Laufbahntheorie (Lent, Brown \& Hackett, 1994, 2001) betont den Stellenwert von Lernerfahrungen und Selbstwirksamkeitserwartungen. Letztere beziehen sich auf das Vertrauen in die eigenen Fähigkeiten, eine Tätigkeit erfolgreich auszuführen (Bandura, 1977, 1986), unabhängig davon, ob institutionelle oder strukturelle Gegebenheiten die Erfolgsaussicht einschränken. Dabei wird die familiäre Herkunft als soziale Umwelt berücksichtigt, die Lernerfahrungen bereit stellt und dadurch die Entwicklung von Interessen begünstigt, aber auch die Umsetzung von Interessen in (Berufswahl-)Ziele und deren Realisierung moderiert. Psychologische Modelle zur Erklärung von Laufbahnentscheiden bieten den Vorteil, dass sie auch Erwartungen bezüglich subjektiv antizipierter Folgen wie Status, Anerkennung oder Selbstverwirklichung (Bandura, 1977, 1986) empirisch überprüfbar machen (Eccles, 1983; Gottfredson, 1981, 1996; Lent et al., 1994, 2001). 


\section{Studien- und Berufswahlmotive von angehenden \\ Lehrpersonen}

Es gibt eine grosse Zahl an Untersuchungen, die die Studien- und Berufswahlmotive von Lehramtsstudierenden bzw. Lehrpersonen analysieren. Berufsbiographische Untersuchungen zeichnen Karrieren von Lehrpersonen nach und ergründen ihre Berufsmotive. Dabei werden in der Regel Motivnennungen ausgezählt oder faktoranalytisch zusammengefasst (Benninghoff \& Müller, 2005; Brühwiler \& Spychiger, 1997; Herzog, Herzog, Brunner \& Müller, 2007). Aus den meisten Studien geht eine Dominanz intrinsischer Motive hervor: Lehrpersonen nennen primär die Freude an der Arbeit mit Kindern und Jugendlichen, die Lernprozessbegleitung und die Zusammenarbeit mit anderen Menschen als Gründe für ihre Berufswahl. Sie zeichnen sich ferner durch eine offene Persönlichkeit mit kommunikativen, sozialen und häufig künstlerischen Interessen aus (für eine Übersicht vgl. Rothland, 2011). Die Mehrheit dieser Untersuchungen beschränkt sich auf (angehende) Lehrpersonen; damit sind allerdings keine Aussagen dazu möglich, inwiefern sich diese von (zukünftigen) Studierenden mit anderen Berufs- und Studienwünschen unterscheiden. Dazu müssten breitere Schichten von Studienberechtigten, etwa Kohorten von Absolvierenden von Gymnasien, mit einbezogen werden. Entsprechende Untersuchungen identifizieren selbstselektive Mechanismen bei der Studienwahl Lehramt. So zeigen beispielsweise Studierende an pädagogischen Hochschulen ein im Vergleich zu anderen Studierenden geringeres Interesse an Wissenschaft (Heine, Spangenberg, Schreiber \& Sommer, 2005; Nagy, 2006; Trautwein et al., 2006). Abele (2011) findet beim Vergleich zwischen Absolvierenden der Mathematik mit Diplom und solchen mit einem Staatsexamen des Lehramts Unterschiede in beruflichen Selbstwirksamkeitserwartungen, in der Karriereorientierung sowie in Zielen bezüglich der Vereinbarkeit von Beruf und Familie, was sich später auf den Berufserfolg auswirkt. Auch andere Untersuchungen verweisen auf systematische Unterschiede zwischen Lehrpersonen und Studierenden mit einer anderen Studienwahl. So weist etwa Nieskens (2009) in ihrer Untersuchung zur Passung zwischen Merkmalen von potentiellen Studierenden und dem Lehrberuf u.a. eine geschlechtsspezifisch geprägte Disposition nach.

Bis anhin existieren wenige Studien zu den Unterschieden zwischen Studierenden der Primar- und der Sekundarstufe I. In diesen Studien zeigt sich, dass Studierende der Primarstufe stärker pädagogisch interessiert sind und die Freude am Umgang mit Kindern betonen, während Studierende der Sekundarstufe I über ein höheres fachliches Interesse verfügen (Brookhart \& Freemann, 1992; Ulich, 1998). Analog zu den stufenspezifischen Unterschieden sowie gestützt auf das Kriterium der optimalen Passung (Holland, 1997) vermuten wir, dass bei angehenden Lehrkräften der Sekundarstufe I - in Abhängigkeit des präferierten Anspruchsniveaus des zukünftigen Schultyps - entweder das fachliche Interesse oder die pädagogisch-didaktische Orientierung im Vordergrund stehen. Wie Studien zur professionellen Kompetenz von Lehrkräften (Kunter et al., 2011) 
zeigen, ist es insbesondere das Zusammenspiel fachlicher und pädagogischer Fähigkeiten, das zum Erleben eigener Kompetenz führt und dadurch auch die berufliche Zufriedenheit positiv beeinflusst.

Weiter lassen sich herkunftsspezifische Aspekte bei der Selbstselektion finden: Lehramtsstudierende - mit Ausnahme des Gymnasiallehramts - kommen seltener aus einem akademischen Elternhaus als andere Studierende (Bathke, Schreiber \& Sommer, 2000; Denzler \& Wolter, 2009; Heine, Spangenberg \& Sommer, 2006; Kühne, 2006; Trautwein et al., 2006). Für die eigene Studie vermuten wir, dass auch herkunftsbedingte Statusaspirationen sowie ökonomisches Kapital dazu führen, dass Maturandinnen und Maturanden aus Akademikerfamilien eine höhere Präferenz für ein universitäres Studium sowie eine höhere Wahrscheinlichkeit für die Umsetzung einer entsprechenden Studienwahl aufweisen. Bei geringeren verfügbaren ökonomischen Ressourcen sollte gemäss Rational-Choice-Ansätzen die Wahrscheinlichkeit steigen, dass sich jemand für ein kürzeres und direkt berufsbefähigendes Studium an einer Fachhochschule bzw. einer pädagogischen Hochschule entscheidet.

Bei der vorliegenden Untersuchung, die sich mit der Frage beschäftigt, wer eine Lehramtsausbildung für die Sekundarstufe I wählt, gilt es folglich, neben Interessen und anderen motivationalen Faktoren Merkmale der sozialen Herkunft sowie institutionell bedingte Aspekte der Ausbildung wie Studiendauer oder direkte Berufsqualifikation zu berücksichtigen. Folgende Fragen stehen dabei im Zentrum:

1. Wer selektioniert sich in Studiengänge der pädagogischen Hochschulen?

2. Zeigen sich bei den angehenden Lehramtsstudierenden unterrichtsstufenspezifische Muster in Bezug auf ihre fachliche und pädagogische Orientierung, ihre Interessen und Laufbahnvorstellungen? Welche Unterschiede lassen sich zwischen Studierenden des Studiengangs Primarstufe und jenen der Sekundarstufe I beobachten?

3. Wie unterscheiden sich Maturandinnen und Maturanden mit einer Präferenz für ein Lehramtsstudium von solchen, die ein universitäres Fachstudium anvisieren?

4. Welche Personengruppen streben eine an die Ausbildung anschliessende Weiterqualifikation an?

\section{Methodisches Vorgehen}

Zur Untersuchung der Studienwahl Lehramt wurden in einem Querschnittsdesign drei verschiedene Stichproben verwendet (vgl. Tabelle 1). Zum einen wurden Maturandinnen und Maturanden an Schweizer Gymnasien zur intendierten Studienwahl befragt (Teilstudie 1), zum anderen wurden Studierende der PH Zürich kurz nach Studieneintritt zu ihrem Studienwahlprozess, ihren 
berufsbezogenen Überzeugungen sowie ihren Laufbahnperspektiven befragt (Teilstudie 2). ${ }^{3}$

\section{Tabelle 1: Teilstudien, Stichproben und Befragungszeitpunkte}

\begin{tabular}{|l|l|l|l|l|}
\hline Teilstudie 1 & Stichprobe 1 & $\begin{array}{l}\text { Repräsentative Zufallsstichprobe } \\
\text { (mehrstufige Cluster): } \\
\text { 1454 Maturanden und Maturandinnen }\end{array}$ & $\begin{array}{l}\text { 9 Deutsch- } \\
\text { schweizer } \\
\text { Kantone }\end{array}$ & $\begin{array}{l}\text { Frühjahr } \\
2006\end{array}$ \\
\hline Teilstudie 2 & Stichprobe 2 & $\begin{array}{l}\text { Vollerhebung: } \\
\text { 186 Studienanfänger/-innen an der } \\
\text { PH Zürich } \\
\text { (Primarstufe inkl. Vorschulstufe und } \\
\text { Sekundarstufe I) }\end{array}$ & PH Zürich & $\begin{array}{l}\text { Herbst } \\
2006\end{array}$ \\
\cline { 2 - 5 } & Stichprobe 3 & $\begin{array}{l}\text { Vollerhebung } \\
\text { 70 Studienanfänger/-innen an der } \\
\text { PH Zürich } \\
\text { (Sekundarstufe I) }\end{array}$ & PH Zürich & $\begin{array}{l}\text { Herbst } \\
2007\end{array}$ \\
\hline
\end{tabular}

Der Fragebogen, der für beide Teilstudien weitgehend identisch gestaltet war, erfasst das gymnasiale Maturitätsprofil, Zeugnisnoten, Motive und Ziele bei der Studien-sowie der Berufswahl, die angestrebte Ausbildung und der Berufswunsch (nur Teilstudie 1), soziodemographische Angaben und (für Teilstudie 2) eine allfällige berufliche Vorbildung. Die Studien- und Berufswahlmotive wurden in Anlehnung an Fiechter, Stienen und Bühler (2004), Niggli (2004), Oesterreich (1987) und Terhart, Czerwenka, Ehrich, Jordan und Schmidt (1994) mit geschlossenen Fragen erhoben und faktoranalytisch zu theoretisch begründbaren Konstrukten (wie Wissenschaftsorientierung, Familienorientierung oder soziale Orientierung) verdichtet (Denzler \& Wolter, 2008). Die Studierenden der PH Zürich (Teilstudie 2) wurden zusätzlich zu ihren subjektiven Theorien über Fähigkeiten und Eigenschaften, die eine erfolgreiche Lehrperson auszeichnen, zu studienbezogenen Selbstwirksamkeitserwartungen (Jerusalem \& Schwarzer, 1986) sowie zu ihren Laufbahnperspektiven befragt.

Bei der Datenanalyse wurden die offenen Antworten inhaltsanalytisch kategorisiert, für die statistische Überprüfung von Gruppenvergleichen wurden $\mathrm{Chi}^{2}$-Tests und T-Tests durchgeführt. Zur Vorhersage der Studienwahl wurden multinomiale logistische Regressionsmodelle gerechnet (Wooldridge, 2002). Als Kovariaten dienten das Geschlecht, die akademische Ausbildung des Vaters ${ }^{4}$ sowie Angaben zum Fächerprofil im Gymnasium. Mit dem Fächerprofil werden einerseits institutionelle Effekte des Bildungssystems berücksichtigt. Da die Fächer (u.a. auch) in Abhängigkeit der kognitiven Fähigkeiten gewählt werden (SBF, 2008), kann andererseits mittels des Profils und der Noten die schulische Leistung näherungsweise erfasst werden. Noten stellen zudem eine wichtige Basis für die subjektiv eingeschätzte Erfolgswahrscheinlichkeit von Ausbildungen dar. Diese wiederum erweist sich als zentral bei Bildungsentscheidungen. Mit den Kovariaten Zeitpräferenz (bezogen auf die Präferenz für eine kurze Ausbildung) 
sowie Risikoaversität werden - in Anlehnung an Rational-Choice-Ansätze wichtige Aspekte der antizipierten Kostenstruktur im Hinblick auf die Wahl eines Studiengangs erfasst.

\section{Ergebnisse}

\section{Ausbildungswah L}

Die statistischen Analysen der Teilstudie 1 zur Vorhersage der Präferenz für ein Lehramtsstudiums erfolgten mittels eines multinomialen logistischen Modells ${ }^{5}$, wobei die an einem Lehramtsstudium interessierten Studierenden nach Unterrichtsstufe differenziert mit angehenden Studierenden verglichen werden, die keine Lehrpersonenausbildung anstreben. Wir bestimmen damit, mit welcher Wahrscheinlichkeit - unter der Kontrolle der gegebenen Faktoren - das Lehramtsstudium auf einer bestimmten Unterrichtsstufe im Vergleich zu einer bestimmten Referenzgruppe mit anderen Studienabsichten angestrebt wird.

Die Resultate der Regressionsanalysen sind in Tabelle 2 dargestellt. Die Studierenden, die sich nicht für eine Lehrpersonenausbildung interessieren, bilden die Referenzgruppe (Modell 1). Dadurch können die drei verschiedenen Lehramtskategorien Primarstufe (inkl. Lehrkräfte der Vorschulstufe), Sekundarstufe I, Sekundarstufe II (Gymnasium) mit Studierenden, die andere Studiengänge präferieren, verglichen werden. Ein Blick auf die Koeffizienten zeigt, dass sich die an einem Studium für die Primarstufe interessierten Studierenden am stärksten von den Maturandinnen und Maturanden mit einem anderen Studienwunsch unterscheiden. Die an einem Lehramtsstudium der Sekundarstufe II interessierten Personen unterscheiden sich kaum von anderen Maturandinnen und Maturanden. Angehende Lehramtsstudierende der Sekundarstufe I unterscheiden sich von ihren Mitschülerinnen und Mitschülern dadurch, dass sie seltener aus einem akademischen Elternhaus stammen und weniger häufig zu den Besten im Fach Deutsch zählen. Ferner weisen sie eine höhere Zeitpräferenz auf; sie äussern häufiger den Wunsch nach einer kurzen Ausbildungsdauer als andere angehende Studierende. Zudem zeichnen sie sich durch ein höheres Bedürfnis nach Sicherheit im Sinne einer erhöhten Risikoaversität aus. Die Ergebnisse der Regressionsanalyse verweisen darauf, dass sich mehr Gemeinsamkeiten als Unterschiede zwischen angehenden Studierenden der Primar- und Sekundarstufe I beobachten lassen.

Die Ergebnisse des zweiten Modelles (siehe Tabelle 2) verweisen ebenfalls darauf, dass sich zwischen den angehenden Studierenden der Primar- und Sekundarstufe I keine signifikanten Unterschiede ergeben. Die zweite Schätzung (vgl. Modell 2 in Tabelle 2) deutet darauf hin, dass der eigentliche Unterschied zwischen angehenden Lehramtsstudierenden für die Volksschulstufe und Studierenden mit einer anderen Studienwahl liegt. Die Gruppe der Studierenden mit einer Affinität zum Lehramtsstudium auf der Primarstufe liegt dabei einfach noch etwas weiter entfernt vom Durchschnitt. ${ }^{6}$ 
Tabelle 2: Determinanten der Berufswahl Lehramt nach Unterrichtsstufen (Multinomiale logistische Regression)

\begin{tabular}{lcccccc} 
& \multicolumn{3}{c}{ MODELL 1 } & \multicolumn{3}{c}{ MODELL 2 } \\
& (Referenzgruppe: Nicht-Lehrpersonen) & \multicolumn{2}{c}{ (Referenzgruppe: Sekundarstufe I) } \\
& PS & S1 & S2 & PS & S2 & N-L \\
\hline Geschlecht: Frau & 1.17 & .84 & -.13 & .33 & -.97 & -.84 \\
& $(.63)+$ & $(.86)$ & $(.56)$ & $(1.08)$ & $(1.03)$ & $(.86)$ \\
Vater Akademiker & -.57 & -1.38 & -.10 & .81 & 1.28 & 1.38 \\
& $(.49)$ & $(.57)^{*}$ & $(.43)$ & $(.72)$ & $(.72)+$ & $(.57)^{*}$ \\
Profil Sprachen & \multicolumn{1}{c}{ Referenzkategorie } & \multicolumn{7}{c}{ Referenzkategorie } \\
Profil Math./NW & -.58 & 1.13 & -.57 & -1.71 & -1.70 & -1.13 \\
& $(.63)$ & $(1.03)$ & $(.58)$ & $(1.09)$ & $(1.25)$ & $(1.03)$ \\
Profil Wirtschaft/Recht & .66 & .73 & -.13 & -.07 & -.87 & -.73 \\
& $(.65)$ & $(.72)$ & $(.40)$ & $(.84)$ & $(.64)$ & $(.72)$ \\
Profil Musik/Gestalten & 1.02 & .62 & -.16 & .40 & -.78 & -.62 \\
& $(.39)^{*}$ & $(.44)$ & $(.40)$ & $(.55)$ & $(.54)$ & $(.44)$ \\
Note Dt., oberstes Dezil & -1.47 & -2.63 & -.07 & 1.16 & 2.56 & 2.63 \\
& $(.53)^{* *}$ & $(.98)^{*}$ & $(.51)$ & $(1.10)$ & $(1.12)^{*}$ & $(.98)^{*}$ \\
Wissenschaftsorientierung & -.58 & -.33 & -.13 & -.26 & .19 & .33 \\
& $(.16)^{* *}$ & $(.23)$ & $(.16)$ & $(.29)$ & $(.25)$ & $(.23)$ \\
Familienorientierung & .56 & .32 & .14 & .25 & -.17 & -.32 \\
& $(.23)^{*}$ & $(.27)$ & $(.19)$ & $(.26)$ & $(.35)$ & $(.27)$ \\
soziale Orientierung & .97 & .71 & .26 & .26 & -.45 & -.71 \\
& $(.25)^{* *}$ & $(.23)^{* *}$ & $(.15)+$ & $(.34)$ & $(.32)$ & $(.23)^{* *}$ \\
Zeitpräferenz hoch & .73 & .64 & -.22 & .09 & -.87 & -.64 \\
& $(.13)^{* *}$ & $(.21)^{* *}$ & $(.22)$ & $(.20)$ & $(.26)^{* *}$ & $(.21)^{* *}$ \\
Risikoaversität & .21 & .39 & .43 & -.18 & .03 & -.39 \\
& $(.22)$ & $.15)^{*}$ & $(.15)^{* *}$ & $(.23)$ & $(.18)$ & $(.15)^{*}$ \\
Konstante & -4.96 & -4.80 & -2.92 & -.16 & 1.89 & 4.80 \\
& $(.61)^{* *}$ & $(.83)^{* *}$ & $(.52)^{* *}$ & $(.98)$ & $(.93)^{*}$ & $(.83)^{* *}$ \\
\hline F(33,8) & & $12.34^{* *}$ & & & $12.34^{* *}$ & \\
N & & 1341 & & & 1341 &
\end{tabular}

Survey multinomiale logistische Regression; Standardfehler (in Klammern) sind für die Clusterstichprobe bereinigt; Gewichtung nach sampling probability

Signifikanzniveau: $+\mathrm{p}<.1,{ }^{*} \mathrm{p}<.05,{ }^{* *} \mathrm{p}<.01$

Legende: PS: Primarstufe; S1: Sekundarstufe I; S2: Sekundarstufe II; N-L: Nicht-Lehrpersonen

Der markante Unterschied von Aspirantinnen und Aspiranten für die Sekundastufe I gegenüber solchen des Gymnasiallehramts sowie anderer Studiengänge besteht in der sozialen Herkunft, in der schulischen Leistung im Fach Deutsch sowie in der Gegenwartspräferenz. Das Ergebnis stützt eine Rational-Choice-Erklärung: Die Herkunft aus einer Nichtakademikerfamilie kombiniert mit der grösseren Gegenwartspräferenz erklärt, weshalb diese Maturandinnen und Maturanden sich tendenziell eher für eine kürzere und direkt berufsbefähigende Ausbildung zur Lehrperson auf Sekundarstufe I interessieren als für ein universitäres Fachstudium mit anschliessender Ausbildung zur Lehrkraft für die Sekundarstufe II. 
Erstwahl versus Zweitwahl nach Studienabbruch

Wie die Auswertung der Daten der Teilstudie 2 zeigt, haben 23.3\% der Lehramtsstudierenden ( 58 von 250) bereits einen Studienabbruch hinter sich. Für fast ein Viertel ist die Ausbildung zur Lehrperson somit eine Zweitwahl. Dabei zeigen sich weder Differenzen nach Geschlecht noch nach Unterrichtsstufe.

Die überwiegende Mehrheit $(86 \%, n=49)$ der Lehramtsstudierenden mit Studienabbruch hatte ein Universitätsstudium- oder ein Studium an einer eidgenössischen technischen Hochschule (ETH) abgebrochen; nur gerade sieben Personen hatten ein Fachhochschulstudium aufgenommen und wieder abgebrochen. Es zeigen sich keine Geschlechterdifferenzen, hingegen Unterschiede nach Unterrichtsstufen: Während die Studierenden der Sekundarstufe I mit Studienabbruch fast alle (97\%) ein Universitäts- oder ETH-Studium abgebrochen hatten, sind es bei den Studierenden der Primarstufe mit 73\% überzufällig weniger mit einem solchen Studienverlauf $\left(\chi^{2}(2)=6.653, p=.036\right)$. Dies lässt sich auch durch die unterschiedliche Vorbildung erklären: Unter den Studierenden der Primarstufe an der PH Zürich beträgt der Anteil Studierender ohne gymnasiale Maturität $28 \%$ und ist damit doppelt so gross wie bei den Studierenden der Sekundarstufe I $\left(\chi^{2}(1)=7.344, p=.007\right)$. Für die Studierenden ohne Maturitätsabschluss ist ein Universitäts- oder ETH-Studium nur über eine Aufnahmeprüfung zugänglich. Die Vorbildung strukturiert die Wahl der Unterrichtsstufe an der PH Zürich somit ein Stück weit vor. Selbst nach einem abgebrochenen Erststudium bleibt dieses Muster bestehen: Wer ein Universitätsoder ETH-Studium abbricht und als Zweitstudium ein Lehramtsstudium wählt, entscheidet sich eher für die Sekundarstufe I; wer ein Fachhochschul-studium abbricht und an die PH Zürich wechselt, entscheidet sich tendenziell für die Primarstufe.

Wie die Auswertung der selbst genannten Gründe für den Abbruch des Erststudiums zeigt, ist die Passung zwischen den eigenen Interessen, Fähigkeiten und dem Studium bzw. späteren Beruf zentral. Als Grund für den Studienabbruch geben die Studierenden an, das Studienfach habe sie inhaltlich doch nicht so interessiert wie ursprünglich angenommen. Ebenso häufig werden auch das Verhältnis von Theorie und Praxis, die Organisation des Studiums sowie fehlende oder unpassende Berufsperspektiven erwähnt, beispielsweise «keine beruflichen Perspektiven ausser Forschung» oder "weil ich später nicht nur Biologie unterrichten möchte». Der Praxisbezug scheint vor allem den Frauen und Männern, die sich für die Primarstufe entschieden haben, wichtig zu sein. Sie geben als Grund für den Studienabbruch vermehrt an, das Studium sei «zu praxisfern» gewesen. Einige Studierende bekunden Mühe mit dem Studiensystem und Studienalltag an einer grossen Hochschule. Ein Befragter meinte beispielsweise: «Ich fühlte mich verloren, da es unorganisiert war und wir so viele Studierende waren». 


\section{Perspektiven nach dem Studienabschluss}

Vor dem Hintergrund theoretischer Überlegungen zur Bedeutsamkeit der subjektiven Einschätzung von Kosten, Nutzen und Erfolgsaussicht bei Bildungsentscheiden sowie der Notwendigkeit, den Nachwuchs im Lehrberuf zu sichern, interessiert die Frage, inwiefern Lehramtsstudierende verschiedener Unterrichtsstufen ihre Bildungsaspirationen auf ihren Studienabschluss (und somit auf die praktische Berufsbefähigung als Lehrer/-in) richten oder ob sie bereits zu Beginn des Studiums die Absicht äussern, ihren Bachelor- oder Master-Abschluss für eine direkt an ihr Studium anschliessende Weiterqualifikation zu nutzen.

Drei Viertel der befragten Lehramtsstudierenden (Teilstudie 2) geben an, direkt im Anschluss an ihre Ausbildung eine feste Stelle als Lehrer/-in suchen zu wollen. Ein knappes Viertel der Befragten ( $\mathrm{n}=59,22.9 \%)$ plant hingegen keinen direkten Berufseinstieg, sondern eine Auszeit (dies nur bei 12 Personen mit dem Ziel, eine Weiterbildung zu absolvieren).

Insgesamt äussert rund ein Fünftel der 256 Studierenden $(21.8 \%, n=56)$ die Absicht, sich direkt nach der Ausbildung weiter qualifizieren zu wollen. Bezüglich der Absicht zur direkten Weiterqualifikation zeigen sich keine Zusammenhänge mit dem Geschlecht $\left(\chi^{2}(1)=.208, \mathrm{p}=.649\right)$, der angestrebten Unterrichtsstufe $\left(\chi^{2}(1)=.002, \mathrm{p}=.964\right)$ und der Vorbildung (Maturitätsabschluss versus andere Vorbildung; $\left.\chi^{2}(1)=.551, \mathrm{p}=.458\right)$. Auch sind es nicht häufiger Studierende aus einem akademischen Elternhaus ${ }^{7}$, die eine direkte Weiterqualifikation anstreben $\left(\chi^{2}(1)=1.251, p=.263\right)$. Allerdings zeigt sich hier ein Interaktionseffekt mit der Vorbildung. Werden nur Studierende ohne Maturitätsabschluss betrachtet, so sind Personen aus einem akademischen Elternhaus in der Gruppe jener, die sich direkt weiterzubilden beabsichtigen, übervertreten $\left(\chi^{2}(1)=5.704, \mathrm{p}<.01\right)$. Bei den Studierenden mit Maturitätsabschluss weicht die Anzahl Weiterbildungswilliger aus einem akademischen Elternhaus nicht überzufällig von jenen ohne ein solches Elternhaus ab. Studierende, die über ein abgebrochenes universitäres Hochschulstudium an die PH Zürich gelangten, sind unter den Studierenden, die eine direkte Weiterqualifikation beabsichtigen, übervertreten $\left(\chi^{2}(1)=3.849\right.$, $\mathrm{p}=.050$ ). Sie möchten jedoch mehrheitlich nicht ein auf ein klassisches Schulfach der Volksschule aufbauendes Studium absolvieren, sondern ziehen Weiterbildungen oder ein Studium im sozialen, pädagogischen oder psychologischen Bereich in Betracht.

Zwischen Studierenden der Primar- und der Sekundarstufe I zeigen sich Unterschiede, wenn die offenen Antworten zur anvisierten Weiterqualifikation analysiert werden. Die Studierenden der Sekundarstufe I geben am häufigsten an, nach Abschluss ihres Lehramtsstudiums in ein universitäres Fachstudium (z.T. mit dem Ziel höheres Lehramt) oder in eine Weiterbildung zur Berufsfachschullehrperson für allgemeinbildenden Unterricht einsteigen zu wollen. An zweiter Stelle folgt der Wunsch nach einer Weiterqualifizierung im Bereich Pädagogik, Psychologie oder Logopädie. Bei den Studierenden der Primarstufe ist eine Weiterbildung im pädagogisch-psychologischen Bereich am beliebtesten, 
gefolgt von der Absicht, zusätzlich zur anvisierten Unterrichtsstufe die Unterrichtsberechtigung für die Sekundarstufe I oder für die Grund-/Basisstufe zu erwerben. Der Wunsch nach einem Stufenumstieg ist damit auf beiden Stufen prominent vertreten, auf der Sekundarstufe I ist er jedoch ausschliesslich auf die nächst «höhere» Schulstufe, d.h. auf die Sekundarstufe II gerichtet.

\section{Selbstwirksamkeit und Anforderungen an das wissen und Können von Lehrpersonen}

Die Studierenden, die ihr Studium bereits begonnen haben, sind relativ zuversichtlich, die Herausforderungen zu meistern. Studierende der Sekundarstufe I verfügen jedoch über eine leicht höhere studiumsbezogene Selbstwirksamkeit als Studierende der Primarstufe $(\mathrm{F}=6.658, \mathrm{p}=.011$, eta $=.033)$. Frauen und Männer unterscheiden sich nicht signifikant.

Um herauszufinden, über welche Vorstellungen Studienanfängerinnen und -anfänger der PH Zürich über das für Lehrpersonen erforderliche Wissen und Können verfügen, wurden sie gebeten, vier Aspekte, die sie selbst als zentral erachten, zu nennen. Die Antworten konnten zu 22 Kategorien zusammengefasst werden. Es zeigen sich keine Geschlechtsunterschiede, hingegen Stufenunterschiede (vgl. Abbildung 1): Lehramtsstudierende auf Sekundarstufe I geben im Vergleich zu angehenden Primarlehrpersonen signifikant häufiger an, Lehrpersonen müssten über Fachwissen und -kompetenz $\left(\chi^{2}(1)=10.196, \mathrm{p}=.001\right)$ sowie Sozialkompetenz und Empathie $\left(\chi^{2}(1)=6.446, p=.011\right)$ verfügen. Demgegenüber messen angehende Primarlehrpersonen den Eigenschaften Kreativität $\left(\chi^{2}(1)=6.080, p=.014\right)$, Offenheit und Flexibilität $\left(\chi^{2}(1)=4.379, \mathrm{p}=.036\right)$ sowie Geduld und Fairness $\left(\chi^{2}(1)=3.915, \mathrm{p}=.048\right)$ eine signifikant grössere Bedeutung zu.

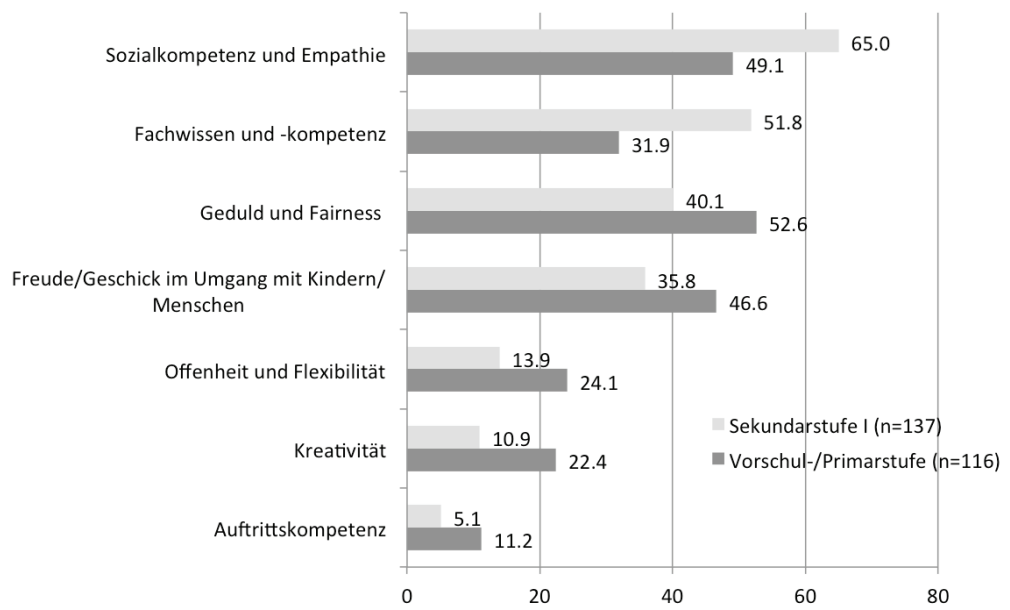

Abbildung 1: Über welches Wissen / welche Fähigkeiten sollten Lehrpersonen verfügen? (Mehrfachantworten, Angaben in Prozent) 


\section{Faz it}

Die an einem Lehramtsstudium für Sekundarstufe I interessierten Studierenden stellen eine heterogene Gruppe dar. Im Vergleich zu Maturandinnen und Maturanden mit einer anderen Studienwahl stammen sie seltener aus einem akademischen Elternhaus, zählen weniger oft zu den Besten im Fach Deutsch und weisen eine Präferenz für ein kürzeres Studium auf. Interessentinnen und Interessenten der Primarstufe unterscheiden sich kaum signifikant von jenen der Sekundarstufe I, hingegen unterscheiden sie sich von denjenigen, die das Gymnasiallehramt (Sekundarstufe II) anstreben. Insgesamt finden wir nicht primär Unterschiede zwischen den Lehrämtern auf der Primar- und Sekundarstufe I, sondern zwischen angehenden Volksschullehrkräften und anderen Studierenden (inkl. angehenden Gymnasiallehrkräften). Die relevanten Unterschiede manifestieren sich folglich zwischen Studierenden einer universitären und einer pädagogischen Hochschule.

Wird die Gruppe der Studienanfängerinnen und -anfänger der PH Zürich betrachtet, so fällt auf, dass Studierende der Sekundarstufe I häufiger über eine gymnasiale Matur verfügen, ein höheres fachwissenschaftliches Interesse sowie eine leicht höhere, auf die Bewältigung der Anforderungen des Studiums bezogene Selbstwirksamkeit aufweisen und Fach- und Sozialkompetenz sowie Empathie als wichtiger, Persönlichkeitseigenschaften wie Kreativität, Offenheit, Flexibilität, Geduld und Fairness aber als weniger wichtig für ihren Beruf einschätzen als Studierende der Primarstufe. Angehende Sekundarlehrpersonen planen zudem häufiger ein universitäres Fachstudium nach Abschluss ihres Lehramtsstudiums als Studierende, die sich für die Primarstufe entschieden haben.

Zusammenfassend lässt sich konstatieren, dass angehende Lehrpersonen der Sekundarstufe I stärker fachorientiert, angehende Lehrpersonen der Primarstufe stärker pädagogisch interessiert sind. Dieses Ergebnis deckt sich mit anderen Studien (Brookhart \& Freemann, 1992; Ulich, 1998). Die Unterschiede in den Präferenzen widerspiegeln sich ein Stück weit in den Anforderungen und der Ausrichtung der verschiedenen Lehramtsstudiengänge. Da bei den angehenden Sekundarlehrpersonen das präferierte Anspruchsniveau (Grundansprüche, erweiterte Ansprüche) nicht erhoben wurde, bleibt allerdings offen, inwieweit die einheitliche Ausbildung von Lehrpersonen der Sekundarstufe I den spezifischen Bedürfnissen und Erwartungen der unterschiedlichen Anspruchsniveaus gerecht wird.

Die Studie macht deutlich, dass die erste Entscheidung von Maturandinnen und Maturanden bei der Studienwahl dem Hochschultyp gilt, und dass dieser Entscheid weniger von Interessen und motivationalen Orientierungen geleitet, sondern stärker von der sozialen Herkunft geprägt ist. Es kommt zu einer Selbstselektion, in der nach sozialer Herkunft unterschiedliche Kosten-Nutzen-Abwägungen wirksam werden. Dies hat zur Folge, dass in der Gruppe der Lehramts- 
studierenden Studierende aus Akademikerfamilien untervertreten sind. Die Gruppe angehender Lehrpersonen ist jedoch in sich relativ heterogen, was ihre Interessen und Erwartungen bezüglich des Studiums anbelangt. Die stärkere fachliche und weniger ausgeprägte pädagogisch-didaktische Orientierung der angehenden Sekundarlehrpersonen sowie ihr höheres Vertrauen in die eigenen Fähigkeiten zur Bewältigung des Studiums werfen die Frage auf, inwiefern diese Gruppe die Option eines Fachstudiums ausserhalb der Lehramtsstudiengänge aufgrund ihrer Herkunft möglicherweise zu schnell verwirft bzw. verworfen hat. Unter der Annahme, dass eine möglichst optimale Passung zwischen den eigenen Interessen und Fähigkeiten und den Anforderungen eines Berufs für eine stabile Studien- und Berufswahl zentral ist, wäre dies für den Verbleib im Lehrberuf kritisch. Studien zum professionellen Lehrerhandeln legen nahe, dass sowohl in der Phase des Berufseinstiegs als auch aus einer längerfristigen Berufsperspektive das Zusammenspiel fachlicher und pädagogischer Kompetenzen unerlässlich ist. Aus Sicht der pädagogischen Hochschulen wäre daher zu überlegen, wie die Wahrnehmung des Lehrberufs als gleichsam fachlich und pädagogisch-didaktisch anspruchsvolle Tätigkeit gefördert und dadurch vermehrt Studierende gewonnen werden könnten, die bereits bei Studienbeginn eine hohe Affinität für beide Bereiche mitbringen. Eine starke Fachorientierung in Kombination mit einem hohen pädagogischen Interesse bzw. entsprechenden Kompetenzen in diesem Bereich sind für das Unterrichten von Schülern und Schülerinnen der Schulstufe mit Grundansprüchen von besonderer Relevanz. Es ist zu vermuten, dass die befragten angehenden Sekundarlehrpersonen mit ihrer stärker fachlichen und weniger ausgeprägten pädagogisch-didaktischen Orientierung eher denjenigen Studierenden entsprechen, die vor der Reform der Lehrerinnen- und Lehrerbildung die Sekundarlehrerausbildung wählten und anschliessend leistungsstärkere Schüler und Schülerinnen unterrichteten. Da diese Studierenden in der Tendenz ein geringeres Interesse am Unterrichten von Schülerinnen und Schülern der Schulstufen mit Grundansprüchen aufweisen, führt dies - bei gleichen oder ähnlichen Anstellungsbedingungen - zu einem Mangel an Lehrpersonen in diesem Bereich. Für die Ausbildung lassen sich daraus folgende Konsequenzen ziehen: Die Ausbildung an den pädagogischen Hochschulen kann einen wichtigen Beitrag leisten, indem sie die Studierenden für die Schülerinnen und Schüler der Klassen mit Grundansprüchen sensibilisiert und der Binnendifferenzierung im Studium ein hohes Gewicht beimisst. Das enge Zusammenspiel zwischen Fächern in den Bereichen Bildung und Erziehung sowie den Fachdidaktiken ist dabei zentral. Die Analyse überfachlicher Lehrund Lernprozesse unter Berücksichtigung der Motivation der Lernenden ist von hoher Bedeutung für angehende Lehrkräfte, die Schülerinnen und Schüler mit vergleichsweise geringeren Bildungschancen und entsprechenden beruflichen Perspektiven unterrichten. In der fachdidaktischen Ausbildung rückt damit die Evaluation bestehender Lernsettings (z.B. in Lehrmitteln) sowie die Entwicklung und Überprüfung kompetenzorientierter Lernaufgaben für Schülerinnen und 
Schüler in heterogenen Leistungsgruppen in den Vordergrund. Dabei geht es nicht darum, stereotype Vorstellungen bezüglich verschiedener Leistungsgruppen zu zementieren, sondern diese in der Praxis kritisch zu reflektieren. Mit einer verstärkten Durchlässigkeit zwischen den Studiengängen können zudem aus dem Pool von Primarlehrpersonen zusätzliche Lehrpersonen für Sekundarklassen mit Grundansprüchen gewonnen werden. Die bereits bestehenden Weiterqualifikationsmöglichkeiten zur Sekundarlehrperson, die Lehrkräften mit einem anderen Diplom offen stehen, könnten entsprechend weiter entwickelt werden. Schliesslich gilt es, Veränderungen auf der gesellschaftlichen und bildungspolitischen Ebene im Auge zu halten und sich der Herausforderung zu stellen, auch in Zukunft kompetente Lehrpersonen für Jugendliche mit weniger günstigen Lernvoraussetzungen zu gewinnen.

\section{Anmerkungen}

1 Die Volksschule umfasst in der Schweiz i.d.R. 6 Jahre Primarschule und 3 Jahre Sekundarstufe I. Die Sekundarstufe I (7.-9. Schuljahr) wird nach Anspruchsniveaus differenziert: Grundansprüche (früher Realschule), erweiterte Ansprüche (früher Sekundarschule), Progymnasium. Das Gymnasium (10.-12. Schuljahr) ist Teil der Sekundarstufe II und zählt nicht mehr zur Volksschule.

2 Für die Zulassung zum Medizinstudium ist nebst einer eidgenössisch anerkannten Maturität ein Aufnahmeverfahren zu bestehen, bei dem gewisse Grundanforderungen zusätzlich geprüft werden.

3 Im Gegensatz zur Stichprobe 1 beinhalten Stichproben 2 und 3 auch Personen ohne gymnasiale Matur, die mittels Aufnahmeverfahren in die PH Zürich eingetreten sind (20\% der Stichproben 2 und 3). Die schulische Vorbildung wie auch das Alter sind deshalb deutlich heterogener als bei Stichprobe 1 .

4 Ausbildung und berufliche Position der Mutter korrelierten stark mit den entsprechenden Variablen des Vaters und hatten, ebenso wie der Migrationsstatus, keine grosse zusätzliche Erklärungskraft. Diese Kovariaten wurden deshalb im bereinigten Modell nicht verwendet.

5 Bei der multinomialen logistischen Regression muss berücksichtigt werden, dass bestimmte für die Analyse relevante Zellen nicht zu klein werden. Die der Analyse zugrunde liegenden Regressionen basieren auf einer repräsentativen Zufallsstichprobe mit 1341 Beobachtungen. Da die erwarteten Zellhäufigkeiten ferner nicht unter $n=5$ fallen, und keine Nullhäufigkeiten in den Randverteilungen auftreten, können wir von einer unverzerrten Teststatistik ausgehen (Andress, Hagenaars \& Kühnel, 1997, S. 204).

6 Es könnte sein, dass sich bei einer grösseren Stichprobe u.U. ein paar weitere Unterschiede zwischen den beiden Kategorien Primarstufe und Sekundarstufe I finden liessen. Das würde aber nichts am Befund ändern, dass die Unterschiede zwischen diesen beiden Stufen deutlich geringer sind als gegenüber dem Lehramt Sekundarstufe II sowie den Nicht-Lehrpersonen.

7 Mind. 1 Elternteil mit einem Abschluss (Lizentiat, Doktorat) einer Universität oder der ETH. 


\section{Literatur}

Abele, A. E. (2011). Prädiktoren des Berufserfolgs von Lehrkräften. Zeitschrift für Pädagogik, $57(5), 674-694$.

Andress, H.-J., Hagenaars, J. A. \& Kühnel, S. (1997). Analyse von Tabellen und kategorialen Daten. Log-lineare Modelle, latente Klassenanalyse, logistische Regression und GSK-Ansatz. Berlin: Springer.

Bandura, A. (1977). Self-efficacy: Toward a unifying theory of behavioral change. Psychological Review, 84 (2), 191-215.

Bandura, A. (1986). Social foundations of thought and action: A social cognitive theory. Englewood Cliffs, NJ: Prentice-Hall.

Bathke, G.-W., Schreiber, J. \& Sommer, D. (2000). Soziale Herkunft deutscher Studien-anfänger: Entwicklungstrends der 90er-Jahre. Hannover: HIS.

Benninghoff, F. \& Müller, K. (2005). Motivations des candidates à l'enseignement. In SRED (Éd.), Gestion prévisionnelle des enseignants (pp. 39-101). Genève: SRED.

Boudon, R. (1973). L'inégalité des chances. La mobilité sociale dans les sociétés industrielles. Paris: Hachette.

Boudon, R. (1987). The individualistic tradition in sociology. In J. C. Alexander (Ed.), The micro-macro link (pp. 45-70). Berkeley: University of California Press.

Bourdieu, P. \& Passeron, J.-C. (1970). La reproduction. Eléments pour une théorie du système d'enseignement. Paris: Editions de Minuit.

Breen, R. \& Goldthorpe, J. H. (1999). Explaining educational differentials. Towards a former rational action theory. Rationality and Society, 9 (3), 275-305.

Brookhart, S. M. \& Freemann, D. J. (1992). Characteristics of entering teacher candidates. Review of Educational Research, 62 (1), 37-60.

Brühwiler, C. \& Spychiger, M. (1997). Subjektive Begründungen für die Wahl des Lehrberufes. Beiträge zur Lehrerbildung, 15 (1), 49-58.

Coleman, J. S. (1994). A rational choice perspective on economic sociology. In N. J. Smelser \& R. Swedberg (Ed.), The handbook of economic sociology (pp. 166-182). York: Russel Sage Foundation.

Criblez, L. (2010). Die Reform der Lehrerinnen- und Lehrerbildung in der Schweiz seit 1990: Reformprozesse, erste Bilanz und Desiderata. In H. Ambühl \& W. Stadelmann (Hrsg.), Tertiarisierung der Lehrerinnen- und Lehrerbildung (S. 22-58). Bern: EDK.

Dawis, R. V. \& Lofquist, L. H. (1984). A psychological theory of work adjustment. Minneapolis: University of Minnesota Press.

Denzler, S. \& Wolter, S. C. (2008). Selbstselektion bei der Wahl eines Lehramtsstudiums: Zum Zusammenspiel individueller und institutioneller Faktoren. Beiträge zur Hochschulforschung, 30 (4), 112-141.

Denzler, S. \& Wolter, S. C. (2009). Sorting into teacher education. How the institutional setting matters. Cambridge Journal of Education, 39 (4), 423-441.

Eccles, J. S. (1983). Expectancies, values, and academic behaviors. In J. T. Spence (Ed.), Achievement and achievement motives (pp. 75-146). San Francisco: Freeman.

Eccles, J. S. (1994). Understanding women's educational and occupational choices: Applying the Eccles et al. model of achievement-related choices. Psychology of Women Quarterly, 18 (4), 585-609.

EDK. (2008). Lehrberuf: Analyse der Veränderungen und Folgerungen für die Zukunft. Bern: EDK.

Erikson, R. \& Jonsson, J. O. (1996). Can education be equalized? The Swedish case in comparative perspective. Boulder: Westview Press.

Esser, H. (1993). Soziologie. Allgemeine Grundlagen. Frankfurt am Main: Campus.

Fiechter, U., Stienen, A. \& Bühler, C. (2004). Zukünftige Lehrpersonen: Berufswahl als pragmatisch orientierte Individualisierung. Eine Studie zur Situation im Kanton Bern. Bern: Institut für Lehrerinnen- und Lehrerbildung. 
Gottfredson, L. S. (1981). Circumscription and compromise: A developmental theory of occupational aspirations. Journal of Counseling Psychology Monograph, 28 (6), 545-579.

Gottfredson, L. S. (1996). Gottfredson's theory of circumscription and compromise. In D. Brown \& L. Brooks (Ed.), Career choice and development (pp. 179-232). San Francisco: Jossey-Bass.

Heine, C., Spangenberg, H., Schreiber, J. \& Sommer, D. (2005). Studienanfänger in den Wintersemestern 2003/2004 und 2004/2005. Wege zum Studium, Studien-und Berufswahl, Situation bei Studienbeginn. Hannover: HIS.

Heine, C., Spangenberg, H. \& Sommer, D. (2006). Studienberechtigte 2004 - Übergang in Studium, Ausbildung und Beruf: Ergebnisse der Befragung der Studienberechtigten 2004 ein halbes Jahr nach Schulabgang im Länder-und Zeitvergleich. Hannover: HIS.

Herzog, W., Herzog, S., Brunner, A. \& Müller, H.-P. (2007). Einmal Lehrer, immer Lehrer? Eine vergleichende Untersuchung der Berufskarrieren von (ehemaligen) Lehrpersonen. Bern: Haupt.

Holland, J. (1997). Making vocational choices: A theory of vocational personalities and work environments. Odessa: Psychological Assessment Resources.

Jerusalem, M. \& Schwarzer, R. (1986). Selbstwirksamkeit. In R. Schwarzer (Hrsg.), Skalen zur Befindlichkeit und Persönlichkeit (S. 15-28). Freie Universität Berlin.

Kühne, S. (2006). Das soziale Rekrutierungsfeld der Lehrer. Zeitschrift für Erziehungswissenschaft, 9 (4), 617-631.

Kunter, M., Baumert, J., Blum, W., Klusmann, U., Krauss, S. \& Neubrand, M. (2011). Professionelle Kompetenz von Lehrkräften. Ergebnisse des Forschungsprogramms COACTIV. Münster: Waxmann.

Lent, R. W., Brown, S. D. \& Hackett, G. (1994). Toward a unifying social cognitive theory of career and academic interest, choice and performance. Journal of Vocational Behavior, 45, 79-122.

Lent, R. W., Brown, S. D. \& Hackett, G. (2001). The role of contextual supports and barriers in the choice of math/science educational options: A test of social cognitive hypotheses. Journal of Counseling Psychology, 48 (4), 474-483.

Leutwyler, B. (2007). Perspektiven im Lehrberuf - ein Diskussionsbeitrag. Beiträge zur Lehrerbildung, 25 (1), 102-112.

Luhmann, N. \& Mayntz, R. (1973). Personal im öffentlichen Dienst - Eintritt und Karrieren. Baden-Baden: Nomos.

Nagy, G. (2006). Berufliche Interessen, kognitive und fachgebundene Kompetenzen: Ihre Bedeutung für die Studienfachwahl und die Bewährung im Studium. Dissertation, Freie Universität Berlin.

Nieskens, B. (2009). Wer interessiert sich für den Lehrerberuf - und wer nicht? Berufswahl im Spannungsfeld von subjektiver und objektiver Passung. Göttingen: Cuvillier.

Niggli, A. (2004). Welche Komponenten reflexiver beruflicher Entwicklung interessieren angehende Lehrerinnen und Lehrer? - Faktorenstruktur eines Fragebogens und erste empirische Ergebnisse. Schweizerische Zeitschrift für Bildungswissenschaften, 26 (2), 343-362.

Oesterreich, D. (1987). Die Berufswahlentscheidung von jungen Lehrern. Berlin: MPIB.

Rothland, M. (2011). Warum entscheiden sich Studierende für den Lehrerberuf? Interessen, Orientierungen und Berufswahlmotive angehender Lehrkräfte im Spiegel der empirischen Forschung. In E. Terhart, H. Bennewitz \& M. Rothland (Hrsg.), Handbuch der Forschung zum Lehrerberuf (S. 268-295). Münster: Waxmann.

SBF. (2008). Evaluation der Maturitätsreform 1995 (EVAMAR): Schlussbericht zur Phase II. Zürich: SBF/EDK.

Super, D. E. (1980). A life-span, life-space approach to career development. Journal of Vocational Behavior, 16, 282-298. 
Terhart, E., Czerwenka, K., Ehrich, K., Jordan, F. \& Schmidt, H. J. (1994). Berufsbiographien von Lehrern und Lehrerinnen. Frankfurt am Main: Peter Lang.

Trautwein, U., Maaz, K., Lüdtke, O., Nagy, G., Husemann, N., Watermann, R. \& Köller, O. (2006). Studieren an der Berufsakademie oder an der Universität, Fachhochschule oder Pädagogischen Hochschule? Zeitschrift für Erziehungswissenschaft, 9 (3), 393-412.

Ulich, K. (1998). Berufswahlmotive angehender LehrerInnen. Die deutsche Schule, 90, 64-78. Wooldridge, J. M. (2002). Econometric analysis of cross section and panel data. Cambridge MA: MIT press.

Schlagworte: Studienwahl, Lehramtsstudium, Sekundarstufe I, Selbstselektion und Motivation für den Lehrberuf

\section{Qui opte pour une formation à l'enseignement secondaire I? Le choix des études et ses liens avec l'origine sociale, la vocation pédagogique et l'intérêt pour les contenus de l'enseignement}

\section{Résumé}

Cette contribution vise à apporter quelques réponses à la question de savoir qui opte pour une formation à l'enseignement et quelles sont les différences en la matière entre les futurs enseignants du primaire et ceux du secondaire. Elle montre qu'il existe des différences entre détenteurs de maturité, soit ceux qui s'intéressent à une carrière dans l'enseignement et les autres, ceci du point de vue de l'origine sociale, des résultats scolaires (en allemand langue maternelle) et de la longueur des études choisies. En outre, par rapport aux futurs enseignants du primaire, les futurs enseignants du degré secondaire se caractérisent par un intérêt plus marqué pour les disciplines scolaires et un intérêt moindre pour les questions pédagogiques.

Mots-clés: Choix des études, études pour devenir enseignant, secondaire I, autosélection et motivation pour la profession de l'enseignement 


\section{Chi sceglie una formazione per insegnare nel secondario I? La scelta degli studi alla luce dell'origine socio-economica, degli interessi pedagogici e dell'orientamento disciplinare}

\section{Riassunto}

Questo articolo si interroga sul profilo di chi sceglie di iniziare un percorso di studio come insegnante nella scuola secondaria inferiore (scuola media), e se sia possibile individuare una differenza tra le persone che studiano per diventare insegnanti nei settori primario e secondario I. I risultati mostrano che chi sceglie un percorso per l'insegnamento si distingue dagli altri maturandi in quanto a origine socio-economica, rendimento scolastico (nota in Tedesco L1) e preferenze temporali. Inoltre, le persone che si formano come insegnanti nel secondario I dimostrano un orientamento disciplinare più forte e un più scarso interesse pedagogico rispetto alle persone che si formano come insegnanti nel settore primario.

Parole chiave: Scelta degli studi, formazione dei docenti, secondario I, autoselezione e motivazione all'insegnamento

\section{Who opts to study lower secondary teaching? Career-choice in light of socio-economic background, educational interest and subject centred orientation}

\section{Summary}

This paper focuses on the self-selection into teacher education studies and investigates the differences between prospective students choosing between primary teaching and lower secondary teaching. The results show that high school graduates interested in teacher education differ from other high school graduates in their socio-economic background, their academic performance (grades in German) and their time preference. Furthermore, students who choose lower secondary teaching are more interested in subject specific factors (content), while students who choose primary teaching are more student-centered.

Keywords: Career choice, teacher education, lower secondary, self-selection and teaching motivation 